\title{
THE ABSOLUTE MAGNITUDES OF THE RR LYRAE STARS
}

\section{JOINT MEETING OF COMMISSIONS \\ $24,27,30,33$ AND 37}

(Edited by W.S. Fitch) 


\title{
THE ABSOLUTE MAGNITUDES OF THE RR LYRAE STARS
}

\author{
SIR RICHARD WOOLLEY \\ Royal Greenwich Observatory, Herstmonceux Castle, Hailsham, England
}

The absolute magnitudes of pulsating variable stars, both RR Lyrae stars and Cepheid variables, may be assessed from observation in three ways: by the classical method of statistical parallaxes, by their occurrence in star clusters whose distance is otherwise known, particularly by ascertaining the position of the main sequence in the HR diagram, and by the Baade-Wesselink method of determining stellar diameters.

As regards the first of these, the method of statistical parallaxes, the RR Lyrae stars lend themselves to this better than do the Cepheid variables, because the velocities relative to the Sun are so much larger. RR Lyrae radial velocities are frequently as high as $200 \mathrm{~km} / \mathrm{sec}$ or even $300 \mathrm{~km} / \mathrm{sec}$, and as many of the stars lie at distances between 1000 and $1500 \mathrm{pc}$ the proper motions of the transverse velocities may be expected to be as high as 0.050 per annum. And, indeed, many investigations have been made recently, among which one may mention those by Plaut, by van Herk, and by the Royal Greenwich Observatory.

The difficulties in the way of a successful attack on the problem start with the necessity to classify the RR Lyrae stars correctly before attempting an analysis. It has been known for some time that the stars are not kinematically homogeneous, the shortest period stars being slower moving, relative to the Sun, than those of the longest periods. In 1953, Pavlovskaya divided the variables into two groups, Group 1 being those with periods less than 0.4 day, and Group 2 being those with periods greater than 0.4 day. The radial velocities of Group 1 stars were substantially smaller than those of Group 2. It is now a question whether the groups should not be further subdivided, in which case suitable criteria must be found, and further, if the groups become too small in number the statistical result becomes unreliable. The kinematic properties are summarized in Table $\mathbf{I}$.

TABLE I

Kinematic properties from radial velocity

\begin{tabular}{llllll}
\hline Pavlovskaya & sub group & $\begin{array}{l}\text { Median } \\
\text { period }\end{array}$ & $\begin{array}{l}\text { Median } \\
|\varrho| \mathrm{km} / \mathrm{sec}\end{array}$ & $\begin{array}{l}\text { Median } \\
\text { latitude }|\mathrm{b}|\end{array}$ & $\begin{array}{c}\text { No. of } \\
\text { stars }\end{array}$ \\
\hline I & Ultra short & $0^{\mathrm{d}} .11$ & 32 & $33^{\circ}$ & 15 \\
& Low amplitude low $\Delta s$ & $0^{\mathrm{d}} .28$ & 56 & $38^{\circ}$ & 18 \\
& Low amplitude high $\Delta s$ & $0^{\mathrm{d}} .31$ & 91 & $54^{\circ}$ & 13 \\
& High amplitude $P_{\text {TRANS }} 0^{\mathrm{d}} .35$ & $0^{\mathrm{d}} .40$ & 26 & $22^{\circ}$ & 25 \\
& & & & & \\
II & $P_{\text {TRANs }} 0^{\mathrm{d}} .44$ & $0^{\mathrm{d}} .49$ & 105 & $39^{\circ}$ & 63 \\
& $P_{\text {TRANS }} 0^{\mathrm{d}} .58$ & $0^{\mathrm{d}} .65$ & 87 & $49^{\circ}$ & 15 \\
\hline
\end{tabular}


Radial velocities are known for about two hundred stars considered to be RR Lyrae type variables, and these vary considerably in accuracy, since some of them are based on only two or three observations of a star whose velocity has a considerable amplitude: but this is not as it happens a major source of error in determining the mean velocity of a sample. The stars have naturally a large dispersion in velocities, the root mean square departure being about one third of the quantity sought (the mean velocity) in the case of the longest period stars, and about two thirds in the case of the short period stars. On this account alone there is a considerable probable error attached to the fundamental assumption that the mean motion of a comparatively small sample of stars is the same whether determined from proper motion or from radial velocity: and in fact we might expect to find on this account a standard error of about $0.2 \mathrm{mag}$. in the mean absolute magnitude of a sample of forty longer period stars, and as much as half a magnitude from a sample of twenty shorter period stars.

This limitation applies to proper motions as much as it does to radial velocities: that is to say that there is an irreducible error in applying the method of statistical parallaxes to a small sample of stars, however well the individual radial velocities and proper motions are known. Nevertheless there are serious and avoidable errors - avoidable at least in principle - which will occur if there are systematic errors in the proper motions. One such source of error has recently been isolated by Dr Clube, who is due to speak later. It resides in the fact that stars whose orbits are somewhat inclined to the plane of the Galaxy lag behind the circular velocity more than do those stars which stay close to the galactic plane (or in other words show a larger Stromberg term). The solar motion relative to these stars is greater by 20 or $30 \mathrm{~km} / \mathrm{sec}$ than it is for the bulk of the nearby stars. Therefore stars seen in the directions of the galactic poles used as standards of reference for RR Lyrae stars will have systematic motions of a kind which will be passed on to the proper motions of RR Lyrae variables unless a correction is made, and this will disturb the statistical parallaxes.

But if we can successfully remove all sources of systematic error in the determination of proper motions - a very big 'if' - the task remains of classifying the variables according to their real kinematic properties, which can be done from radial velocities alone, and then grouping them into sets which have the same absolute magnitudes, without which the method of statistical parallax yields at best a vague general mean. To achieve this, we can seek guidance from the behaviour of the variable stars in globular clusters. The variable stars in globular clusters were classified by Oosterhoff into type I and type II according to the mean period of their Bailey $a$-types, type I having a mean period of 0.55 and type II 0.65 . This idea can be developed by introducing a quantity called $P_{\text {TRANS }}$, the period at which there is a transition from Bailey type $a$ variables with large amplitudes (and longer periods) to Bailey type $c$ with smaller amplitudes and shorter periods. There is a gap between the shortest period $a$ type occurring in a cluster and the longest period $c$ type. This gap is variable but usually amounts to 0 d. $1 . P_{\text {TRANS }}$ is defined as the period of the shortest $a$ type in the cluster and was introduced by Christy in his theoretical work on RR Lyrae models.

Preston's quantity $\Delta s$ has not been determined for cluster variables except in very 
few cases. A similar quantity, or at least an indication of metal abundance, has been developed for the late type giants in globular clusters by Deutsch, and extended by Kinman who classifies clusters as $A, B$ or $C$, where $A$ indicates slightly weak metallic lines, $B$ weak metallic lines and $C$ very weak metallic lines; so that Deutsch's type $A$ corresponds to $\Delta s 0$ to 3 and Deutsch's $C$ corresponds to $\Delta s$ nearly equal to 10 among the nearby stars.

In addition several astronomers, notably Sandage, have estimated the absolute magnitude of the cluster variables by comparison with main sequence stars in the same cluster. This can be compared with a value of $M_{v}$ derived from $P_{\text {TRAns }}$ with the help of Christy's theoretical models.

The results for some clusters are given in Table II.

TABLE II

Absolute magnitudes of RR Lyrae stars in globular clusters

\begin{tabular}{|c|c|c|c|c|c|}
\hline Cluster & Deutsch's class & $P_{\text {TRANS }}$ & $M_{v}$ from & n sequence & $\begin{array}{l}M_{v} \text { from } \\
P_{\text {TRANS }}\end{array}$ \\
\hline M3 & A & $0^{\mathrm{d}} .496$ & +0.28 & \multicolumn{2}{|c|}{ Sandage (Arp) +0.80} \\
\hline M5 & A & $0^{\mathrm{d}} .455$ & +0.6 & Arp & +0.96 \\
\hline M13 & A & - & -0.09 & (Sandage) & - \\
\hline M15 & $\mathrm{C}$ & $0^{\mathrm{d}} .565$ & +0.51 & (Sandage) & +0.57 \\
\hline M92 & $\mathrm{C}$ & $0^{d} .60$ & +0.47 & (Sandage) & +0.46 \\
\hline 47 Tuc & A & - & +0.44 & (Tifft) & - \\
\hline
\end{tabular}

Sandage's results (1964) have been revised (towards truer absolute magnitudes) but the results have not yet been published.

If we now turn back to the field RR Lyrae stars, and wish to investigate their absolute magnitudes by the method of statistical parallaxes, we have to enquire whether we expect them to have the same kinematical properties, whatever the period or other characteristics, and whether we expect them to have the same absolute magnitude. The first question can be answered, at least to some extent, without answering the second, if we confine our attention to radial velocities; but we must have some system of classification, either Pavlovskaya's division into Group I and Group II or something more elaborate.

The next slide 2 shows the period amplitude diagram of all the field variables for which photoelectric values of the $V$ amplitude are known. Open circles show stars for which Preston's spectrum classification $\Delta s$ is 0 to 3, corresponding roughly to Deutsch's type $A$ (slightly weak metal lines) and filled circles show stars with $\Delta s 4$ or greater (Deutsch type $B$ or $C$ ).

The differences between the field stars and the cluster stars which are apparent without any appeal to statistical parallaxes are:

(1) The occurrence of a number of variables with much shorter periods than any which have been found in globular clusters (where the shortest periods so far known 
are about 0.24 ). They have often been discussed and have been classified as $\delta$ Scuti variables or as dwarf Cepheids.

(2) The field has very few $c$ type variables, apart from these, in comparison with the average of the globular clusters.

(3) The field seems to have a class of stars of its own, with amplitudes from 1 to $1 \frac{1}{2}$ magnitudes and periods much shorter than $P_{\text {TRANS }}$ of NGC 6171, namely 0.42 . These stars are exclusively of low $\Delta s$. Almost all the stars with $P>0.44$ have $\Delta s \geqslant 4$ (with only seven exceptions out of 61 stars classified).

The stars with $0.2<P<0$ d 45 and of low amplitude are evenly divided between those with high and low $\Delta s$, and if the stars are divided according to $\Delta s$ the kinematic properties of the two subclasses seem quite different.

The appearance of two values of $P_{\text {TRANS }}$ among the longer period field stars suggests that they should be divided into at least two classes. It is difficult at present to set up a satisfactory discriminant, but however the division is made, there seems to be no striking difference in the kinematical behaviour of the subclasses.

Thus far the arguments, so far as they concern the field stars, have been statistical; but there is a method of determining the absolute magnitude of an individual pulsating star originally proposed by Baade and developed by Wesselink. It has not been widely applied, partly for lack of adequate data and partly on account of lack of confidence in the method.

If $F_{V}$ is the flux of radiation per $\mathrm{cm}^{2}$ contributing to $V$ of a star of radius $R$, the luminosity $L_{V}$ of this star is

$$
L_{V}=4 \pi R^{2} F_{V}
$$

or in practical form

$$
M_{V}-S_{V}+5 \log R=15.15,
$$

where $S_{V}$ is the flux on a magnitude scale and $M_{V}$ the absolute magnitude. If we differentiate this expression with respect to time and remember that $M_{V}$ differs from the apparent magnitude $V$ by a constant we have

$$
\frac{\mathrm{d} V}{\mathrm{~d} t}-\frac{\mathrm{d} S_{V}}{\mathrm{~d} t}=-2.1715 \frac{1 \mathrm{~d} R}{R \mathrm{~d} t},
$$

so that if $\mathrm{d} V / \mathrm{d} t$ is determined from the light curve and $\mathrm{d} R / \mathrm{d} t$ determined from radial velocity measurements, the radius $R$ can be determined if $S_{V}$ is known as a function of the colour (B-V). This is the principle of the method. If this is not known, but if it is assumed that $S_{V}$ is a function of the colour alone, the radius can be determined by taking two points of equal color, one on the rising branch of the light curve and one on the descending branch: then for these two points

$$
\Delta V+5 \Delta \log R=0 \text {, }
$$

from which $R$ can be determined if $\Delta R$ is known from radial velocities. This is the essential improvement to Baade's proposal for determining radii of pulsating stars introduced by Wesselink. 
TABLE III

Radii and absolute magnitudes of Cepheid strip variable stars

\begin{tabular}{|c|c|c|c|c|c|c|}
\hline Star & Investigator & Star $R / R_{\odot}$ & $R / R_{\odot}$ & B-V Fernie & $M_{v}$ Fernie & $\begin{array}{l}M_{v} \text { from } \\
\text { Wesselink's } \\
\text { tables }\end{array}$ \\
\hline \multirow[t]{3}{*}{$\delta$ Cep } & Stebbins & 53 & 49 & 63 & -3.9 & -3.6 \\
\hline & Oke & 40 & & & & \\
\hline & Whitney & 53 & & & & \\
\hline \multirow[t]{3}{*}{$\eta \mathrm{Aql}$} & Stebbins & 68 & 66 & 67 & -4.1 & -4.1 \\
\hline & Oke & 64 & & & & \\
\hline & Whitney & 67 & & & & \\
\hline \multirow[t]{2}{*}{ Y Oph } & $\mathrm{Abt}$ & 72 & 95 & (70) & -5.0 & -4.8 \\
\hline & Becker & 118 & & & & \\
\hline$\kappa \mathrm{Pav}$ & Rodgers & 21 & 21 & (56) & -3.6 & -1.5 \\
\hline$\beta$ Dor & Rodgers & 105 & 105 & (68) & -4.4 & -5.0 \\
\hline l Car & Rodgers & 138 & 138 & 93 & -5.3 & -4.7 \\
\hline \multirow[t]{3}{*}{ RR Lyr } & Stebbins & 7.2 & 7.0 & 29 & +0.7 & +0.5 \\
\hline & Abt & 6.0 & & & & \\
\hline & Oke & 7.8 & & & & \\
\hline SU Dra & Oke et al. & 5.2 & 5.2 & 33 & +0.6 & +0.2 \\
\hline
\end{tabular}

Fernie's formulae use $\log P$ and (B-V) (Astrophys. $J .140,1482,1964)$.

The 'Wesselink' result quoted uses Wesselink's table of $S_{V}$ and the value of $R$ shown as a mean.

Radii have been determined by this method in a number of cases shown in Table III. If the radius is known, it can be used to determine an absolute magnitude if it is supposed that $S_{V}$ can be computed from the colour B-V or from the spectral type. This has usually been done by using an effective temperature, but it can also be done with the help of tables prepared by Wesselink relating $S_{V}$ to B-V. These are based on stellar angular diameters observed by Hanbury Brown with an interferometer.

Fernie adopts a period-radius relation, which leads him to an absolute magnitude depending on $P$ and (B-V). This leads to $R / R_{\odot}=64$ for $\kappa$ Pav, and 5.1 for RR Lyrae, both of which are very different from the results given by the Baade-Wesselink method, and these differences explain the differences in the absolute magnitudes shown for these two stars in the last two columns.

There are many indications that an effect occurs in the rising branch which causes a modification in the relation between specific emission and colour of a kind which necessitates a modification in the Baade-Wesselink method. This was proposed by Abt in 1959 but not yet, so far as I know, widely applied.

It seems quite clear that if the Baade-Wesselink method can be improved and successfully applied to RR Lyrae stars, we shall be able to say with much greater confidence what their absolute magnitudes are, and whether the connexions between $P_{\text {TRANS }}$ and absolute magnitude suggested by theory or by work on globular clusters can be substantiated by the field stars. 


\section{DISCUSSION}

Bok: With the advent of large telescopes in the southern hemisphere, there is a great future for the study of RR Lyrae stars in the Magellanic Clouds.

Woolley: I agree.

Kharadze: Dr. Alania of Abastumani Observatory has been systematically observing the spectra of several RR Lyrae stars, and has shown that $\Delta s$ varies with phase during the pulsation period. Shouldn't one take into account this variation?

Woolley: Kinman takes account of the variation of $\Delta s$ with phase, but not all observers do.

Buscombe: In view of the great difficulty of deriving reliable radii for classical Cepheids, can one in fact achieve the necessary resolution in time and radial velocity from spectrograms of faint RR Lyrae variables with periods less than $8 \mathrm{hr}$ ?

Woolley: Yes, in many cases. 